\title{
One in Place, Another One to Go
}

\section{Jaroslava Halper*}

Department of Pathology, College of Veterinary Medicine, The University of Georgia, Athens, GA 30605-7388, USA

\section{Editorial}

Two papers in this special issue review were selected for publication because of their timely topic. The paper by French and Frazier [1] brings us up to date on the state of the art of our knowledge of basic and applied science for vascular endothelial growth (VEGF). Because VEGF has been considered a specific angiogenic agent, the inhibition of its activity either using antagonists of its signaling pathway or monoclonal antibodies directed against its receptor was thought to be the cornerstone of newly developing specific antitumor strategy. This notion was buttressed by recent findings about integral roles of VEGF singaling in vascular integrity, modulation of extracellular matrix, hypoxic responses, tumor immunoreactivity and stem cell recruitment. All these and many other issues are well covered by French and Frazier The importance of VEGF in tumor biology has been confirmed by studies on overexpression of VEGF in several tumor types and its correlation with poor prognosis [2] and on responsiveness of tumors to anti-VEGF therapy. VEGF-A levels (in particular the $\mathrm{VEGF}_{206}$ isoform) have been demostrated to be increased in mammary, lung, brain, pancreatic, ovarian, kidney, and bladder carcinomas. Moreover, studies on transgenic animals indicate that VEGF acts not only as an angiogenic growth factor but also as an autocrine agent directly stimulating the proliferation of primary tumor cells and the growth of metastatic lesions $[3,4]$.

French and Frazier also emphasize the importance of VEGF-C and VEGFR-3 in lymphangiogenesis, and thus in cancer progression. Newly developed anti-VEGF-C or anti-VEGFR-3 agents are capable of blocking lymphangiogenesis, and thus cancer progression at least in rodent models $[5,6]$. If successful in human clinical trials this would represents a truly novel approach to chemotherapy.

Avastin $^{\odot}$ (bevacizumab), manufactured by Genentech, represents one of the first examples of commercially successful biologicals. This compound is a monoclonal anti-VEGF antibody that has been used in combination with classic chemotherapeutic drugs to treat patients with metastatic carcinoma of the colon or rectum, patients with unresectable, locally advanced, recurrent or metastatic non-squamous non-small cell lung cancer, and patients who have not received chemotherapy for metastatic HER2-negative breast cancer [7]. Though it was withdrawn recently by the FDA from the line of approved drugs for treatment of advanced breast cancer, a couple of recent studies have shown effectiveness of Avastin in treatment of early breast cancer $[8,9]$. The addition of bevacizumab to neoadjuvant chemotherapy led to a statistically significant increase in remission rate of HER2negative breast cancer though this was accompanied by increased rate of side effects, seen in patients treated with anti VEGF agents, such hypertension, left ventricular dysfunction and certain infections in both studies $[8,9]$.

The second paper in this issue raises the awareness of readers for granulins, a less known family of growth factors [10]. Though the first progranulin or PGRN, the so called transforming growth factor e, was first described as a mitogen primarily for epithelial cells back in 1987 [11], the recognition of its significance is only a recent phenomenon. In addition to its mitogenic activity for both epithelial and mesenchymal, it also participates in embryonic development; wound healing, CNS function among others. Though the therapeutic potential is largely unexplored, the overexpression of granulins in several cancers (of breast, ovary, prostate, brain, hepatocellular carcinoma) but not in others (lung, endometrial carcinoma) raises the possibility of proliferation promoting effect of granulins in specific tumors. The assumed role progranulin plays in carcinogenesis is at least partially due to its mitogenicity, therefore potential therapeutic benefit would have to be connected with inhibition of receptor activation or signal transduction. However, relatively little is known about granulin receptor and signal transduction systems, especially in the upstream pathways. That might be a reason why the scientific community lags behind in developing biological therapeutic agents based on granulins. What we know indicates that PGRN binds with sortilin on cell surfaces which is likely to be part of a protein turnover mechanism [12]. PGRN also binds to and inhibits the TNF-receptors, and associates with the Toll-like receptor-9 [10]. These interactions are important in the regulation of inflammation.

The likely unusual signaling pathway suggests that targeting mediators in this pathway might lead to fewer side effects than experienced with other inhibitors directed towards targets within signaling pathways of VEGF, PDGF and EGF where there is a lot of similarities, and therefore crosstalk among molecules, thus resulting in sometimes unexpected side effects. However, new studies show that downstream in the PRGN pathway there is convergence with other signaling pathways, such as activation of the MAPK and PI3K signaling systems, though the details vary with different cell types and tumors. Interestingly, unlike some other growth factors PRGN possesses the ability to initiate mitosis on its own (e.g., in fibroblasts lacking the IGF-1 receptor) [10]. Whether that means that targeting of upstream molecules will be more specific or whether it will lead to modification of downstream mediators is difficult to predict. Perhaps more feasible approach would the disruption of PRGN binding to components of the extracellular matrix, such as perlecan or integrin. Going in a different direction, Park et al. devised a strategy using RNA interference targeting the GRN gene to suppress proliferation of cancerous cells in vitro and also to suppress growth of tumors after transplantation of such into Balb/C nude mice [13].

${ }^{*}$ Corresponding author: Jaroslava Halper, Department of Pathology, College of Veterinary Medicine, The University of Georgia, Athens, GA 30605-7388, USA, Tel: 706-542-5830; Fax: 706-542-5828; E-mail: jhalper@uga.edu

Received February 02, 2012; Accepted February 04, 2012; Published February 06, 2012

Citation: Halper J (2012) One in Place, Another One to Go. J Carcinogene Mutagene S2:e001. doi:10.4172/2157-2518.S2-e001

Copyright: (c) 2012 Halper J. This is an open-access article distributed under the terms of the Creative Commons Attribution License, which permits unrestricted use, distribution, and reproduction in any medium, provided the original author and source are credited. 
It is also possible that disturbances of progranulin signaling pathway play more essential and, perhaps more importantly, specific role in the pathogenesis of certain degenerative CNS diseases than in carcinogenesis. For example, a mutation in a copy of the human GRN gene results in a form of frontotemporal lobar dementia that is characterized by neuronal atrophy in the frontal and temporal cerebral lobes and that usually afflicted middle aged people. Bigni et al. entertain the possibility that specific pharmacological agents can counteract progranulin haploinsufficiency in patients with this type of dementia carrying GRN mutation [14].

Granulin is a growth factor we should pay close attention to in the near future.

\section{References}

1. French KJ, Frazier KS (2012) The role of VEGF and VEGF receptors in carcinogenesis. J Carcinogene Mutagene S4-001.

2. Ferrara N (2005) VEGF as a therapeutic target in cancer. Oncology 3: 11-16

3. Huh Jl, Calvo A, Stafford J, Cheung M, Kumar R, et al. (2005) Inhibition of VEGF receptors significantly impairs mammary cancer growth in C3(1)/Tag transgenic mice through antiangiogenic and non-antiangiogenic mechanisms. Oncogene 24: 790-800.

4. Schoeffner DJ, Matheny SL, Akahane T, Factor V, Berry A, et al. (2005) VEGF contributes to mammary tumor growth in transgenic mice through paracrine and autocrine mechanisms. Lab Invest 85: 608-623.

5. Dong Z, Wei F, Zhou C, Sumida T, Hamakawa H, et al. (2011) Silencing
Id-1 inhibits lymphangiogenesis through down-regulation of VEGF-C in oral squamous cell carcinoma. Oral Oncol 47: 27-32.

6. Yang H, Kim C, Kim MJ, Schwendener RA, Alitalo K, et al. (2011) Soluble vascular endothelial growth factor receptor-3 suppresses lymphangiogenesis and lymphatic metastasis in bladder cancer. Mol Cancer 10: 36.

7. Halper J (2010) Growth factors as active participants in carcinogenesis: a perspective. Vet Pathol 47: 77-97.

8. Bear HD, Tang G, Rastogi P, Geyer CE Jr, Robidoux A, et al. (2012) Bevacizumab added to neoadjuvant chemotherapy for breast cancer. $\mathrm{N}$ Eng J Med 366: 310-320.

9. von Minckwitz G, Eidtmann H, Rezai M, Fasching PA, Tesch H, et al. (2012) Neoadjuvant chemotherapy and bevacizumab for HER2-negative breast cancer. N Engl J Med 366: 299-309.

10. Zhang Y, Bateman A (2012) The glycoprotein growth factor progranulin promotes carcinogenesis and has potential value in anti-cancer therapy. J Carcinogene Mutagene S2-001.

11. Halper J, Moses HL (1987) Purification and characterization of a nove transforming growth factor. Cancer Res 47: 4552-4559.

12. Hu F, Padukkavidana T, Vægter CB, Brady OA, Zheng Y, et al. (2010) Sortilinmediated endocytosis determines levels of the frontotemporal dementia protein, progranulin. Neuron 68: 654-667.

13. Park MY, Park YS, Nam JH (2011) RNA interference against granulin-epithelin precursor prevents hepatocellular carcinoma growth: its application as a therapeutic agent. Int J Oncol 39: 853-861.

14. Bigni B, Premi E, Pilotto A, Padovani A, Borroni B (2012) Disease-Modifying Therapies in Frontotemporal Lobar Degeneration. Curr Med Chem.
This article was originally published in a special issue, Growth factors : Carcinogenesis handled by Editor(s). Dr. Jaroslava Halper, The University of Georgia, USA; Dr. Ian Stuart Zagon, The Pennsylvania State University, USA 